\section{Granulozytäre Antigene}

K. Kleesiek ${ }^{1}$, C. Götting ${ }^{2}$, J. Diekmann ${ }^{3}$, J. Dreier ${ }^{4}$ und M. Schmidt ${ }^{5}$

${ }^{1}$ Ehemaliger Direktor des Instituts für Laboratoriums- und Transfusionsmedizin, Herz- und Diabeteszentrum NordrheinWestfalen Ruhr-Universität Bochum, Bad Oeynhausen, Deutschland

${ }^{2}$ MVZ Labor Limbach Nürnberg GmbH, Nürnberg, Deutschland

${ }^{3}$ Institut für Laboratoriums- und Transfusionsmedizin, Herzund Diabeteszentrum NRW, Bad Oeynhausen, Deutschland ${ }^{4}$ Herz- und Diabeteszentrum Nordrhein-Westfalen; Institut für Laboratoriums- und Transfusionsmedizin, Universitätsklinik der Ruhr-Universität Bochum, Bad Oeynhausen, Deutschland

${ }^{5}$ Institut für Laboratoriums- und Transfusionsmedizin, Universitätsklinik der Ruhr-Universität Bochum, Bad Oeynhausen, Deutschland

\section{Synonym(e) HNA}

Englischer Begriff human neutrophil antigens (HNA)

Definition Antigene auf Granulozyten

Beschreibung Die wichtigsten Antigene auf Granulozyten, die HNA-Antigene, sind häufig verantwortlich für eine
Immunisierung nach Schwangerschaften oder, seltener, nach Bluttransfusionen, wobei bei heutigen Blutpräparaten aufgrund der Leukozytenfiltration die Immunisierungswahrscheinlichkeit gering ist und reine Granulozytenpräparate nur nach gesonderter, eng gefasster Indikation verabreicht werden. Klinisch wichtige Antigene sind die polymorphen Formen HNA-1a, $-1 \mathrm{~b}$ und $-1 \mathrm{c}$ des Fc $\gamma$-Rezeptor IIIb (Fc $\gamma$ RIIIb), einem über Glykosylphosphatidyl-Inositol (GPI) in der Plasmamembran verankerten Glykoprotein, das mit einer Kopienzahl von $2-3 \times 10^{5}$ je Zelle ausschließlich auf neutrophilen Granulozyten exprimiert wird und der Bindung von Immunkomplexen dient. Weitere wichtige Glykoproteinantigene sind das HNA-2a-Antigen (CD177), das in unterschiedlichen Prozentsätzen jeweils nur auf einer Subpopulation der Granulozyten eines Individuums exprimiert wird, und das HNA-3a, beide ebenfalls GPI-verankert. Ebenfalls bedeutsame Granulozytenantigene, wie das HNA-4 (CD11b) und HNA-5 (CD11a), gehören zur Familie der $\beta 2$ Integrine.

\section{Literatur}

Clay ME, Schüler RM, Bachowski GJ (2010) Granulocyte serology: current concepts and clinical significance. Immunohematology 26:11-21

Reil A, Bux J (2015) Geno- and phenotyping of human neutrophils antigens. Methods Mol Biol 1310:193-203 